\title{
IMPROVEMENT AND MONITORING OF SOIL HEALTH
}

Tim Thoden, Leendert Molendijk, Jonny Visser, Gerard Korthals; Applied Plant Research, Wageningen UR, NL-8200 AK Lelystad, Netherlands; e-mail: tim.thoden@wur.nl

\section{Einleitung}

Unter dem Begriff der Bodengesundheit wird allgemeinen die Fähigkeit des Bodens verstanden, „innerhalb gewisser ökologischer und landnutzungstechnischer Grenzen, als vitales System zu funktionieren, ohne dabei die Erfüllung der an ihn gestellten Funktionen (Nutzungsfunktion, Filterfunktion, Erholungsfunktion, etc.) zu vernachlässigen“ (Doran \& Zeiss 2000). Eng damit verbunden ist der Begriff suppressiver bzw. wehrbarer Böden. Sie wurden von Baker \& Cook (1974) als Böden beschrieben, „in denen es trotz des Vorhandenseins eines Pflanzenpathogens, einer anfälligen Wirtspflanze und Krankheit-fördernder klimatischer Bedingungen nur zu wenigen und in der Regel schwachen Krankheitsausbrüchen kommt“. Häufig wird dieses Phänomen mit biologischen Bodenparametern in Verbindung gebracht, die dafür sorgen, dass ein anwesender Pathogen nicht überhand nimmt. Es stellt sich nun die Frage inwieweit gezielte Eingriffe, wie die Einbringung organischen Materials, zu einer Verbesserung der Bodengesundheit beitragen können.

Vor diesem Hintergrund starteten wir 2006 einen langläufigen Praxisversuch, der darauf abzielt, Methoden zu entwickeln, die der Bodengesundheit zuträglich sind. Ferner sollen innerhalb dieses Projekts Indikatoren entwickelt werden, anhand derer klare Aussagen über den aktuellen Zustand der Bodengesundheit abgeleitet werden können. Dabei richtet sich das Hauptaugenmerk auf die individuenreichste Gruppe der Bodenfauna, die Nematoden (Bongers \& Ferris 1999, Wilson \& Kakouli-Duarte 2009). Sie eignen sich aus mehreren Gründen als Indikatororganismen. So sind Nematoden leicht aus dem Boden zu extrahieren, auf Familien- oder Gattungsniveau zu determinieren und aufgrund ihres Vorkommens in verschiedenen trophischen Ebenen (bacteriophage, fungiphage, omnivore, carnivore und phytophage Arten) ein guter Spiegel des Bodennahrungsnetzes.

\section{Methoden}

Die Versuchsfläche in Vredepeel (Limburg, Niederlande) liegt auf einem sandigen Boden, der einen hohen Besatz mit Wurzelläsions-Nematoden (Pratylenchus penetrans) und dem Erreger der Verticillium-Welke (Verticillium dahliae) aufweist. Die ca. ein Hektar große Fläche 
wurde zu Beginn des Versuchs in 16 Streifen $(6$ x 60 m) aufgeteilt (Abb. 1). Diese wurden nachfolgend entweder nach biologischen (8 Streifen) oder konventionellen Verfahren (8 Streifen) bewirtschaftet. Der Anbauplan umfasste dabei in beiden Systemen für diese Region typische Kulturen (2006 = Getreide; 2007 = Kartoffel; 2008 = Lilien; 2009 = Getreide; 2010 = Kartoffel). Ferner wurden seit Versuchsbeginn in jedem der Systeme zweimalig (2006/2007 u. 2009/2010) 10 Bodenbehandlungen durchgeführt, um die Bodengesundheit zu verbessern (Abb. 1). Dabei kamen sowohl biologische, chemische als auch physische Methoden zum Einsatz. Diese umfassten z. B. den Anbau einer Klee-Gras-Mischung oder von Studentenblumen (Tagetes patula), die Biofumigation mit Brassicaceaen oder als Kontrollvariante eine Schwarzbrache. Die Biofumigation wurde mit braunem Senf (Brassica juncea cv. Energy; Aussaatmenge $12 \mathrm{~kg} / \mathrm{ha}$ ) durchgeführt. Dabei wurden die Pflanzen nach 34monatiger Wachstumsphase fein-gehäckselt, $15 \mathrm{~cm}$ tief eingefräßt, die Bodenoberfläche dicht gerollt und abschließend mit 25 mm beregnet. Da der Aufwuchs der Pflanzen im Herbst 2006 schlecht war, wurden bei ihrer Einarbeitung zusätzlich Brokkoli-Abfälle zugefügt. Insgesamt konnten so ca. 40 t/ha an glukosinolathaltigem Material eingearbeitet werden. Die Studentenblume (Tagetes patula cv Ground Control; $10 \mathrm{~kg} / \mathrm{ha}$ ) wurde im Hochsommer ausgesät und die Pflanzenreste im folgenden Frühjahr gehäckselt und auf der Versuchsfläche belassen. Die Kleegras-Mischung (Lolium perenne, Lolium multiflorum, Trifolium repens, Trifolium pratense, $25 \mathrm{~kg} / \mathrm{ha}$ ) wurde ebenfalls im Sommer gesät, über Winter stehen gelassen und dann eingearbeitet.

Alljährlich im Frühjahr wurden bzw. werden innerhalb aller Behandlungen dutzende chemische, physische und biologische Bodenparameter ermittelt. Hierbei wird, neben der kompletten Analyse der mikrobiellen Artenzusammensetzung und deren Aktivität, nicht nur die Anzahl pflanzenparasitärer Nematoden (Pratylenchus) ermittelt, sondern zusätzlich auch die Artenzusammensetzung der kompletten Nematodenfauna erfasst. Dabei wird zunächst das Fraßverhalten (bakteriophage, fungiphage, omnivore, carnivore, phytophage) von 150 Nematoden einer Bodenprobe $(100 \mathrm{ml})$ unter dem Mikroskop ermittelt und diese Tiere anschließend auf Gattungs- bzw. Artniveau bestimmt (Bongers, 1994). Zudem wird jedes Tier nach der von Bongers (1990) entwickelten cp-Skala (competitor-persister) klassifiziert, und auf diesen Werten basierend ökologische Zeigerwerte - wie der Maturity-Index - berechnet. 


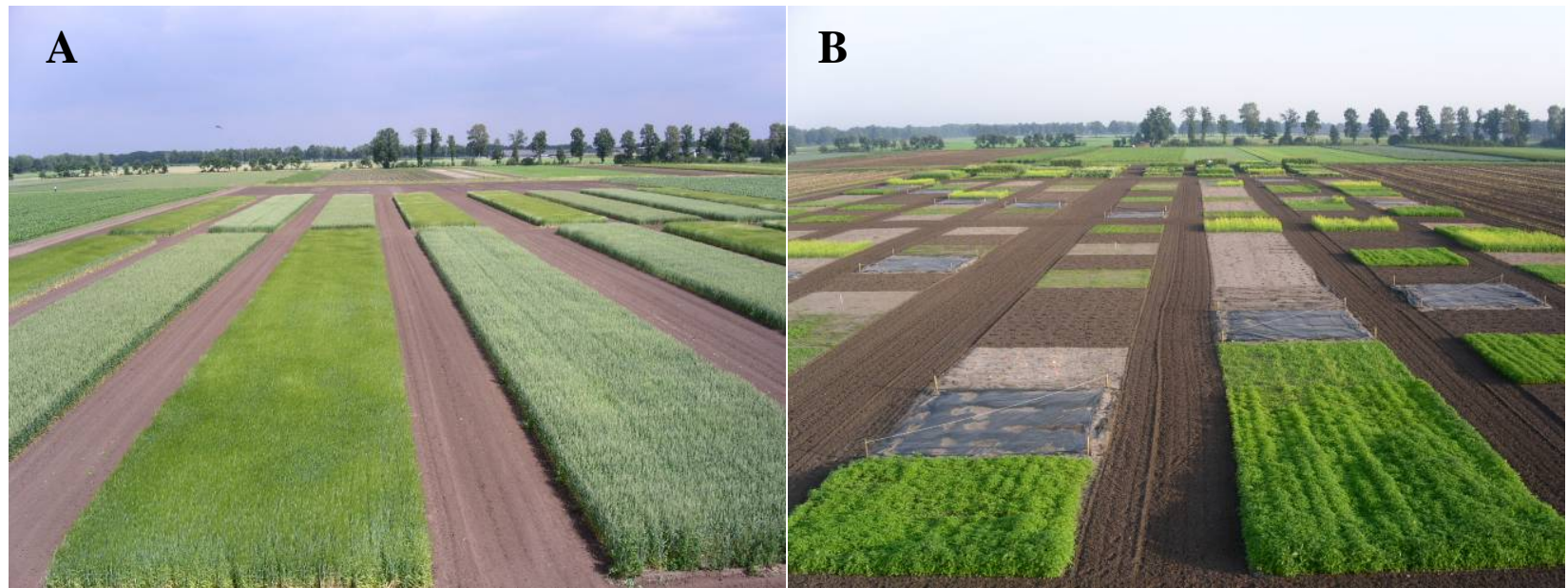

Abbildung 1: (A) Angelegte Versuchsstreifen biologischer und konventioneller Betriebssysteme (4-fach wiederholt); (B) Durchführung von 10 Bodenbehandlungen (per Streifen) (160 Parzellen insgesamt).

\section{Ergebnisse und Diskussion}

Abbildung 2 zeigt, dass die Biofumigationsvariante im Vergleich zur Kontrolle (d. h. natürliche Abnahme bei Schwarzbrache) in beiden Systemen zu keiner statistisch signifikanten Abnahme des Hauptschaderregers führte. Eher schien sich die Besatzdichte post Biofumigation - leicht erhöht zu haben. Dies spricht dafür, dass brauner Senf (B. juncea), wie z. B. schon von Belair et al. (2002) berichtet, eine Wirtspflanze für $P$. penetrans darstellt (s. auch Artikel Korthals et al. in diesem Band). Gleichfalls scheint die eigentliche Biofumigation (Einarbeitung) keine bedeutsamen nematoxischen Effekt hervorzurufen. Im Gegensatz dazu reduzierte der Anbau von T. patula in beiden Systemen Pratylenchus (Abb. 2), was ebenfalls schon länger bekannt ist (Evenhuis et al. 2004). Aus nematologischer Sicht als „indiskutabel“ stellte sich der Anbau der Klee-Gras-Mischung dar, der auf diesen sandigen Böden zu einer explosionsartigen Vermehrung von Pratylenchus führte (Abb. 2). Diesen Effekt sehen wir auch noch in aktuellen Daten (2009) zurück (Daten nicht präsentiert). 


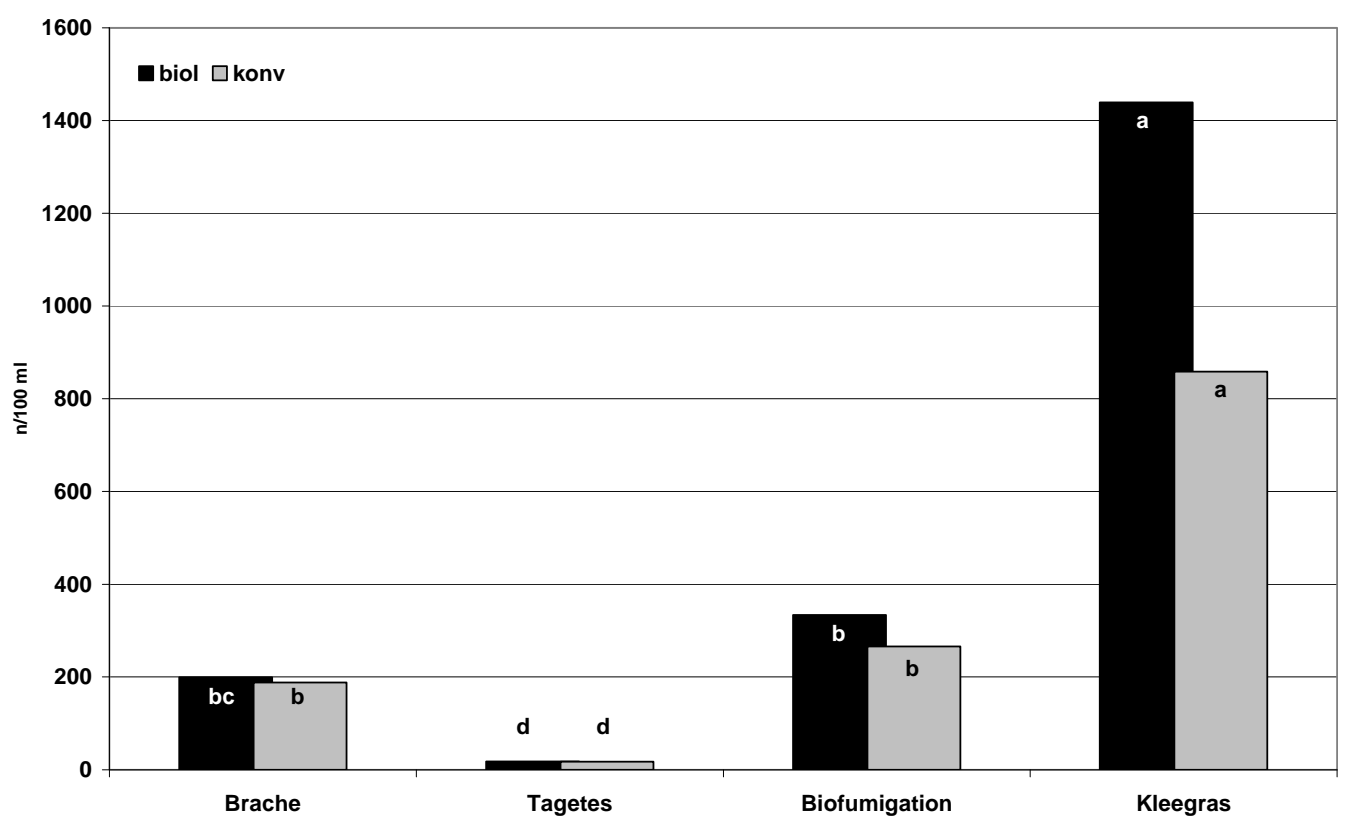

Abbildung 2: Die Besatzdichte mit Wurzelläsions-Nematoden 04/2007 nach einmaliger Durchführung der Bodenbehandlungen (09/2006-03/2007) (Signifikanzniveaus beziehen sich auf das jeweilige Anbausystem).

Signifikante Unterschiede zwischen den Behandlungen lagen auch bzgl. ihres Effekts auf die Abundanzen verschiedener Nematoden-Fraß-Typen vor (Abb. 3). Die Anzahlen phyto- und bakteriophager Nematoden lagen dabei bei Biofumigation im oberen Mittelfeld der hier angegebenen Varianten. Dies zeigt, dass B. juncea nicht nur für Pratylenchus sondern auch für andere phytophage Nematoden Wirtspflanze ist. Ferner stimulierte die Einarbeitung von B. juncea und die damit verbundene Zufuhr organischen Materials die Population bacteriophager Nematoden, deren Anzahl in der Biofumigationsvariante um ca. 1/3 über dem Vergleichswert der Schwarzbrache lag (Abb. 3). Diese Gruppe von Nematoden hat u.a. starken Einfluss auf Mineralisierungsprozesse im Boden und etliche Studien haben gezeigt, dass höhere Anzahlen an bacteriophagen Nematoden zu einer Erhöhung pflanzenverfügbarer Nährstoffe führen (z. B. Ingham et al., 1985). Ebenso scheinen sie positive Effekte auf die Wurzelmorphologie zu verursachen (Mao et al. 2006). Neben der fehlenden Wirkung auf phytoparasitäre Nematoden sind auch diese Daten ein Indiz dafür, dass die Biofumigation keine nematiziden Effekte verursachte. Keine statistisch signifikanten Unterschiede zeigte die Biofumigation bzgl. der anderen Fraßtypen. 
Im Gegensatz zur Biofumigation zeigte der Anbau von Tagetes eine selektive Wirkung gegen phytophage Nematoden. Dabei sollte allerdings angemerkt werden, dass es durchaus Arten phytoparasitärer Nematoden gab, die sich an diesem Gewächs vermehrten (Abb. 3). Schädliche Nebeneffekte auf bacteriophage Arten waren nicht zu erkennen.

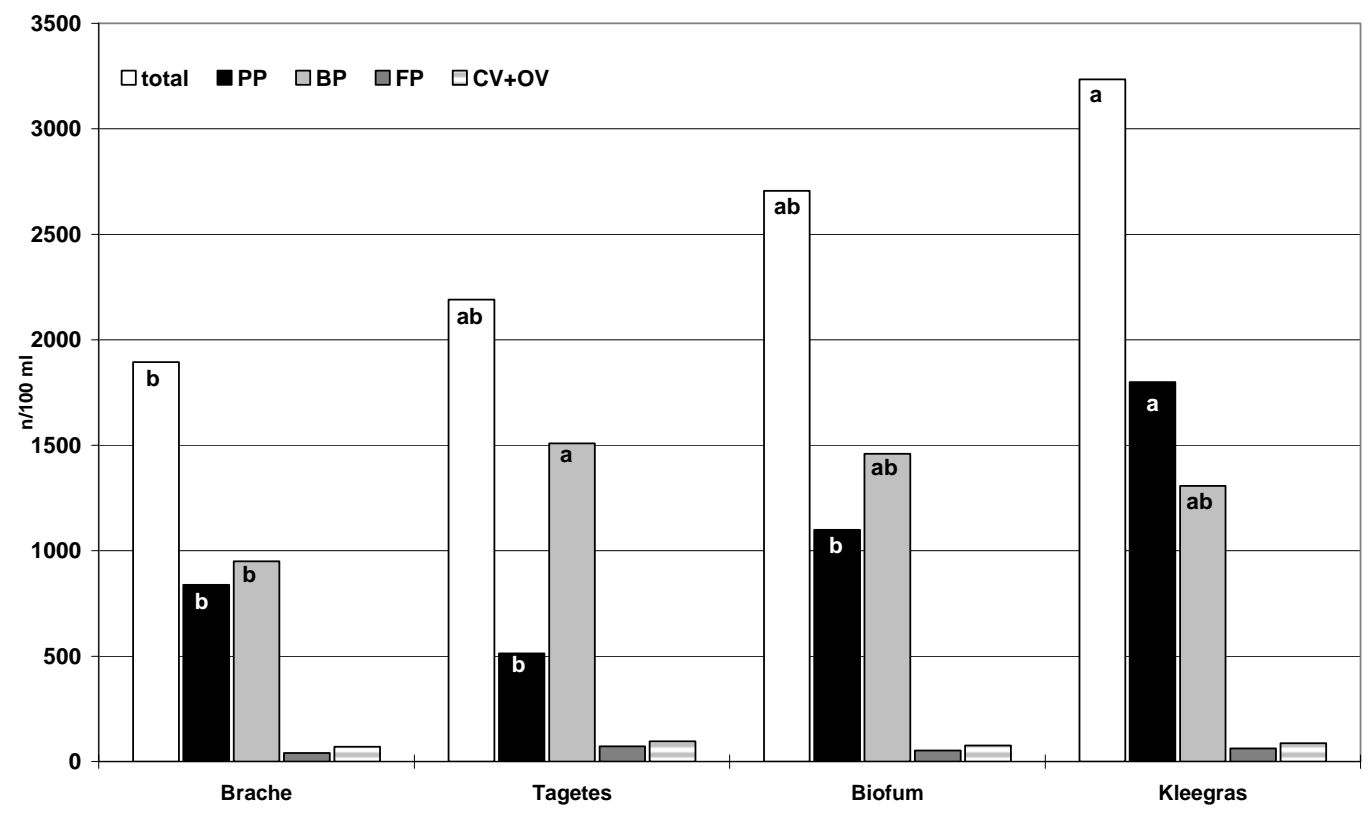

Abbildung 3: Die Abundanz verschiedener Fraßtypen 04/2007 nach einmaliger Durchführung der Boden-behandlungen (Signifikanzniveaus gelten jeweils innerhalb des Fraßtyps) (PP = phytophage; $\mathrm{BP}=$ bakteriophage; $\mathrm{FP}=$ fungiphage; $\mathrm{CV}+\mathrm{OV}=$ carnivore + omnivore) .

Zusammenfassend bleibt festzuhalten, dass wir im Rahmen dieser Untersuchung keine nematizide Wirkung der Biofumigation nachweisen konnten. Dies galt sowohl für phytophage als auch bacteriophage Tiere. Trotzdem ist dieses Verfahren positiv zu bewerten, da es über die Einarbeitung organischen Materials zu einer Stimulierung und Förderung des Bodenlebens kommt.

\section{Literatur}

Belair G., Fournier Y., Dauphinais N., Dangi O.P. (2002). Reproduction of Pratylenchus penetrans on various rotation crops in Quebec. Phytoprotection 83: 111-114.

Bongers T., Ferris H. (1999). Nematode community structure as bioindicator in environmental monitoring. Tree 14: 224-228.

Bongers T. (1994). De Nematoden van Nederland. Publication Foundation Royal Dutch Natural History Society. 
Bongers T. (1990). The maturity index: an ecological measure of environmental disturbance based on nematode-species composition. Oecologica 83: 14-19.

Doran J.W., Zeiss M.R. (2000). Soil health and sustainability: managing the biotic component of soil quality. Applied Soil Ecology 15: 3-11.

Evenhuis A., Korthals G.W., Molendijk L.P.G. (2004). Tagetes patula as an effective catch crop for long-term control of Pratylenchus penetrans. Nematology 6: 877-881.

Ingham R.E., Trofymow J.A., Ingham E.R., Coleman D.C. (1985). Interactions of Bacteria, Fungi, and their Nematode Grazers: Effects on Nutrient Cycling and Plant Growth. Ecological Monographs 55: 119-140.

Mao X.F., Hu F., Griffiths B., Li H.X. (2006). Bacterial-feeding nematodes enhance root growths of tomato. Soil Biology \& Biochemistry 38: 1615-1622.

Wilson M.J., Kaukali-Duarte T. (2009). Nematodes as Environmental Indicators. CABI, Wallingford. 